\title{
Functional measurement scales of painful electric shocks
}

\author{
BILL JONES and MAXWELL GWYNN \\ Carleton University, Ottawa, Ontario, Canada
}

\begin{abstract}
Subjects were required to rate the combined intensities of two factorially paired shocks ranging in intensity from $1 \mathrm{~mA}$ through 2, 3, 4, and $5 \mathrm{~mA}$. In Experiment 1, an anchored 1-20-point rating scale was used, and in Experiment 2, one of two scales, 1-15 points or 1-25 points, was provided to the subject. Data from all three conditions were fit by a weighted average model, $\mathrm{R}_{\mathrm{ij}}=w \mathrm{~s}_{\mathrm{i}}+(1-w) \mathrm{s}_{\mathrm{j}}$, where $\mathrm{R}_{\mathrm{ij}} \mathrm{s}$ are mean ratings, $s_{\mathrm{i}}$ and $\mathrm{s}_{\mathrm{j}}$ are scale values of the first and second shocks, and $w$ is a weight parameter. The derived psychophysical functions were linear in all three cases, and the slope increases with the number of available categories, in conformity to range-frequency theory. In a third experiment, subjects were divided according to the ratings they gave of pain elicited by the 5-mA shock. The weighted averaging model provided an appropriate integration model for both higher and lower pain raters. Higher raters tended to accord an equal weight to both shocks, whereas lower raters accorded greater weight to the second stimulus. Implications for research on analgesic procedures are discussed.
\end{abstract}

Many attempts to have subjects scale the magnitude (or sometimes the "unpleasantness," Ekman, Frankenhaeuser, Levander, \& Mellis (1964) of painful stimuli have been based on the presumption that a psychophysical law, some functional relation between stimulus magnitude and subjective pain, is a necessary precursor to the evaluation, in the laboratory, of analgesic methods (see, e.g., Cross, Tursky, \& Lodge, 1975; Ekman et al., 1964; Gracely, McGrath, \& Dubner, 1978; Stam, Petrusic, \& Spanos, 1981; Sternbach \& Tursky, 1964). Almost all such experiments have relied on either ratings of single stimuli or upon magnitude estimation (Stevens, 1957, 1975) in which the subject is instructed to give a number in proportion to the perceived magnitude of the stimulus (see, e.g., Cross et al., 1975; Gracely et al., 1978; Stam et al., 1981; Sternbach \& Tursky, 1964; Stevens, 1957, 1975; Stevens, Carton, \& Shickman, 1958).

Both ratings and magnitude estimates rest upon the usually unexamined assumption that the numbers given by a subject (or at least the numbers averaged across subjects) have certain important scale properties. For example, researchers who have followed Stevens have almost automatically assumed that magnitude estimates yield ratio scales of subjective magnitudes (see, e.g., Cross et al., 1975; Gracely et al., 1978; Stevens, 1957, 1975; Stevens et al., 1958; Sternbach \& Tursky, 1964), although the reasoning here often appears to be no stronger than the assertion that subjects are capable of following, and in-

This research was made possible by a grant from the Medical Research Council of Canada to the senior author and to N. P. Spanos. The authors' mailing address is: Department of Psychology, Carleton University, Ottawa, Ontario K1S 5B6, Canada. deed do follow, the instruction to give numbers in proportion to perceived magnitudes (cf. Shepard, 1981, p. 30).

On the assumption that equal stimulus ratios do give rise to equal ratios of subjective magnitudes, geometric mean magnitude estimates have been routinely fit by power functions, such as

$$
\mathbf{R}_{\mathbf{i}}=\mathbf{a} \mathbf{I}_{\mathrm{i}}^{\mathbf{b}}+\mathbf{c},
$$

or

$$
\mathbf{R}_{\mathrm{i}}=\mathbf{a}\left(\mathrm{I}_{\mathrm{i}}-\Delta \mathrm{I}\right)^{\mathrm{b}} \text {, }
$$

where the $R_{i} s$ are mean magnitude estimates obtained for associated values of $I_{i}$, the intensity of the stimulus (electric shock data have typically been fit as a function of intensity in milliamps, although Cross et al., 1975, and Jones, 1980, have fit psychophysical data as a function of $I^{2}$, the power of the signal), a is a constant dependent upon scale units, $b$ is a rate parameter, $c$ is an arbitrary constant, and $\Delta I$ is a correction for threshold. Stevens (1957) originally argued that each sensory channel would be associated with a specific value of the exponent, $b$, representing the amplitude of sensory transduction for that channel.

The earliest reported value of $b=3.5$ for electric shock (Stevens et al., 1958; Stevens, 1966, later reported an even higher value of 4.5) fits the notion that the perception of hurtful stimuli is characterized by what Stevens called "a process of expansion." Subjective magnitudes would grow as a positively accelerated function of physical magnitude, and so rapidly afford information about potential tissue damage. Unfortunately other studies have turned up 
much lower values of $b$, typically in the range of 0.70 (Beck \& Rosner, 1968, obtained for Equation 2) to about 2.0. Interestingly, Sternbach and Tursky (1964) found that exponents were actually lower when painful shocks were included in the stimulus range.

Concentration on Equations 1 and 2 may, in fact, have obscured the form of the psychophysical function obtained from magnitude estimates of painful stimuli. McCallum and Goldberg (1975) found that magnitude estimates of shock intensities were as well, and sometimes better, fit by a linear rule as by a power rule. Stam et al. (1981) reached much the same conclusion for data obtained as a function of time when pain was induced in a cold pressor experiment.

A more fundamental question than either the form of the psychophysical function or the variation in the parameters concerns the priority given to the establishment of the psychophysical law. Anderson (e.g., 1981) has consistently argued that power laws of magnitude estimates do not necessarily provide psychophysical laws but, rather, may constitute "an expression of response bias"' (see also Poulton, 1979). He has shown that a sufficient condition for the determination of a psychophysical law is the prior validation of an appropriate psychological law that describes how discrete pieces of information are integrated in a single response. For example, if a subject experiences two electric shocks in a sequence and is required to rate their combined intensity, the rating responses may constitute a weighted average of the subjective magnitudes (the "scale values") of the stimuli. Ignoring error terms and response variability, we may write

$$
\mathbf{R}_{\mathrm{ij}}=\mathrm{ws}_{\mathrm{i}}+(1-\mathrm{w}) \mathbf{s}_{\mathrm{j}},
$$

where the $R_{i j} s$ are mean ratings, $s_{\mathrm{i}}$ and $s_{j}$ are scale values of the first and second stimuli, respectively, and $w$ is a weight parameter reflecting the importance or the salience of the stimulus in the integrated judgment. Validation of Equation 3 leads straightforwardly to the psychophysical function for the given experimental context (Anderson, 1981). The context includes, obviously, the dynamic range and other physical parameters of the stimuli, and also factors such as instructions to the subject, whether or not a standard stimulus is presented and the magnitude of any standard, numerical biases adapted by the subjects, and carry-over effects from previous experiments (see, e.g., Anderson, 1975; Birnbaum, 1978; Mellers \& Birnbaum, 1982; Parducci, 1974; Poulton, 1967, 1979; Robinson, 1976).

\section{EXPERIMENT 1}

This experiment is a test of the generality of Equation 3 and of the psychophysical function,

$$
\overline{\mathrm{X}}_{\mathrm{ij}}=\mathrm{bI}^{2}+\mathrm{c} \text {, }
$$

where the $\bar{X}_{i j} s$ are the means of the row and column marginals for $\mathrm{i}=\mathrm{j}$ or, in other words, the scale values of the stimuli derived from a factorial experiment. In the previous study by Jones (1980), the paired stimulus intensities in the painful condition were 1 , 2,3 , and $4 \mathrm{~mA}$. In this experiment, the paired stimuli will range from 1 through $2,3,4$, and $5 \mathrm{~mA}$. In practice, the psychophysical function will probably depend upon the stimulus range (e.g., Poulton, 1967). A more important aim of this experiment is to establish whether or not the integration model (Equation 3) still holds when the stimuli are experienced as more painful. Previous work (Jones \& Gwynn, 1983) has established that a 5-mA shock is nearly 1.5 times more likely than a 4-mA shock to be rated as intolerably painful, and it is quite possible that an average increase in the painfulness of the shock pairs could disrupt the integration process.

\section{Method}

Subjects. The subjects were 10 undergraduates $(8$ males and 2 females) who participated for course credit after being advised that the experiment would include painful stimuli. The subjects were free to withdraw participation at any time during the experiment without loss of course credit. In response to a brief questionnaire, all the subjects assessed themselves as being in good general health.

Apparatus. Two Lafayette devices, modified to meet C.S.A. standards of safety, delivered ac pulses for a fixed duration of $500 \mathrm{msec}$ through concentric electrodes (Tursky, Watson, \& O'Connell, 1965) into an electric skin circuit that maintained impedance at 5,000 $5500 \Omega$. The duration of the shock and the time interval of $1,000 \mathrm{msec}$ between shocks were controlled by locally constructed equipment.

To minimize extraneous and possibly distracting noise, the subject wore padded industrial ear muffs (Bilsom Viking).

Procedure. The subject was seated in a comfortable armchair, and the two electrodes were attached to the inside surface of the right forearm, one at about $8 \mathrm{~cm}$ from the wrist and the other at about $8 \mathrm{~cm}$ from the elbow. On each trial, a factorial pairing of shock intensities $(1,2,3,4$, and $5 \mathrm{~mA})$ was presented in sequence. The subject was instructed to rate the combined intensity of each pair along a numerical scale from 1 to 20 . Anchor points were provided by instructing the subject to call " 1 " the combined intensity of a pair of shocks each $.75 \mathrm{~mA}$ in intensity and to call " 20 " the combined intensity of a pair of shocks each $5.25 \mathrm{~mA}$ in intensity. The order in which pairings were presented was randomized over trials, and each pair was replicated three times.

\section{Results}

The mean ratings are presented graphically in Figure 1. The curves are roughly parallel and linear. Analysis of variance indicated significant main effects for rows (first stimulus intensities) $[F(4,36)=$ $16.93, \mathrm{p}<.001]$ and columns (second stimulus intensities) $[F(4,36)=19.22, p<.001]$ and the absence of a significant rows $\times$ columns interaction $[F(16,144)=$ $1.36, \mathrm{p}=.17]$. Graphically and statistically, therefore, we have evidence to support the contention that Equation 3 provides an appropriate integration rule for electric shocks in the range 1 to $5 \mathrm{~mA}$. Increasing the painfulness of the shock intensities does not disrupt processing, at least at this global level. 
Figure 2 is a plot of the psychophysical function obtained from the marginal means of the design. The curve appears linear, and a two-parameter linear function provided a superior fit compared with the $\mathbf{I}^{2}$ rule (Table 1 ).

Two other psychophysical rules, Equation 1 and a two-parameter power function omitting the additive constant, c, were also fit to the data in Figure 2. Values of the rate parameter, b, and the goodnessof-fit statistic, $r^{2}$, are shown in Table 1. The linear rule yielded a slightly better fit than the threeparameter power rule.

There was some evidence of a recency effect in that subjects tended to accord greater weight to the second stimulus. The estimated value of $w$ (see Anderson, 1974, for estimation procedures) was 0.38 .

The results of the present experiment support the psychological integration model suggested by Jones (1980). However, we were unable to confirm the hypothesis that psychological scale values of painful electric shock constitute a linear function of the power of the stimulus.

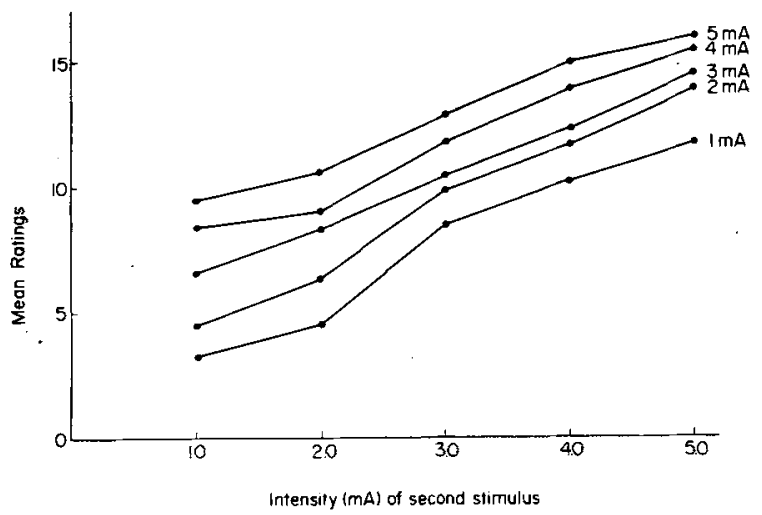

Figure 1. Mean ratings of the first stimulus intensity $(1,2,3$, 4 , and $5 \mathrm{~mA}$ ) as a function of the second stimulus intensity (1-20point rating scale) (Experiment 1).

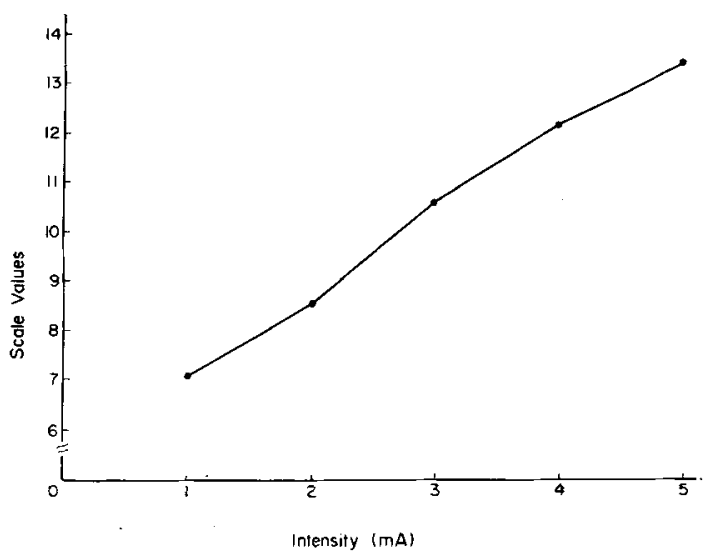

Figure 2. The psychophysical function derived from the rating data in Figure 1.
Table 1

Rate Parameters (b) of the Psychophysical Function,

$X_{i j}=b I+c\left(\right.$ Linear), $X_{i j}=b I^{2}+c\left(I^{2}\right), X_{i j}=a I^{b}$

(Two-Parameter Power) and $X_{i j}=a^{b}+c$

(Three-Parameter Power), Together with Values of $r^{2}$, the Goodness-of-Fit Statistic, for Each Function (Experiment 1)

\begin{tabular}{ccccc} 
& & & \multicolumn{2}{c}{ Power } \\
\cline { 3 - 5 } & Linear & $\mathrm{I}^{2}$ & $\begin{array}{c}\text { Two- } \\
\text { Parameter }\end{array}$ & $\begin{array}{c}\text { Three- } \\
\text { Parameter }\end{array}$ \\
\hline $\mathrm{b}$ & 1.65 & 0.26 & 0.41 & 0.86 \\
$\mathrm{r}^{2}$ & .9955 & .9434 & .9810 & .9905 \\
\hline
\end{tabular}

Note-Marquardt's (1964) algorithm was used to fit the threeparameter power function, and the method of least squares was used to estimate statistics for the remaining functions.

\section{EXPERIMENT 2}

The spacing of the five stimuli in Experiment 1 was approximately linear. However, it may be that the spacing of stimuli is influenced by the number of stimuli in relation to the number of available categories. Poulton (1979) has noted, following Gibson and Tomko (1972), that ratings of single electric shocks in the range 1.5 to $5 \mathrm{~mA}$ were a linear function of $\log I$ when seven categories were supplied, but concave upward when judgments on a 49-point scale were required. The number of response categories in our view is simply part of the context for the psychophysical judgment (see, e.g., Mellers \& Birnbaum, 1982). In the following experiments, therefore, subjects gave ratings of the combined intensity of two shocks, as in Experiment 1, except that one group was provided with a 1-25-point scale and the second group with a 1-15-point scale. These relatively narrow response ranges were chosen because pilot work had established that subjects' ratings on a 1100-point scale tended overwhelmingly to be multiples of 5 or 10; that is, their ratings were effectively on a 10-point or a 20-point scale.

The subjects in the present experiment were 19 undergraduates (15 males and 4 females) who had not been tested in Experiment 1. Nine were assigned at random to the 1-25-point condition, and the remainder, to the 1-15-point condition.

\section{Results and Discussion}

Figure 3 is a plot of the mean ratings for each condition. Both are very similar to the plot illustrated in Figure 1. Analysis of variance for the 1-25-point scale showed significant effects of rows $[F(4,32)=20.57$, $\mathrm{p}<.001]$ and columns $[\mathrm{F}(4,32)=17.82, \mathrm{p}<.001]$, with $F(16,128)<1$ for the interaction. Substantially the same result was obtained for the 1-15-point condition [rows, $F(4,36)=39.63, \mathrm{p}<.001$; columns, $F(4,36)=65.26, p<.001$; and the interaction, $F(16$, 144) $=1.25, \mathrm{p}=.24]$. 
The psychophysical functions for the two conditions are shown in Figure 4. Table 2 gives estimates of rate parameters for various fits of the psychophysical function together with values of $r^{2}$. The linear fit was either superior or only marginally inferior to the three-parameter power fits. Since this was also the case in Experiment 1, we may conclude that the spacing of the five stimuli did not change as the number of available categories was increased or decreased.

It is also clear from Tables 1 and 2 that the slope of the psychophysical function increases monotonically with the number of rating categories. The rate of increase in scale values depends upon the response range. Analogous results have been obtained for magnitude estimates. Mellers and Birnbaum (1982) provided a modulus of 100 and varied the instructions to subjects to include an example of either 300

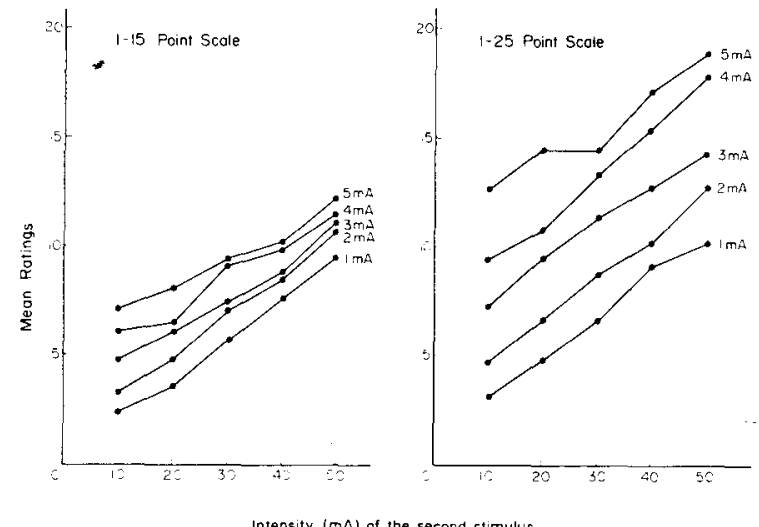

Figure 3. Mean ratings of the first stimulus intensity as a function of the second (Experiment 2). The data for the 1-15-point scale are in the left-hand panel, and the data for the 1-25-point scale are in the right-hand panel.

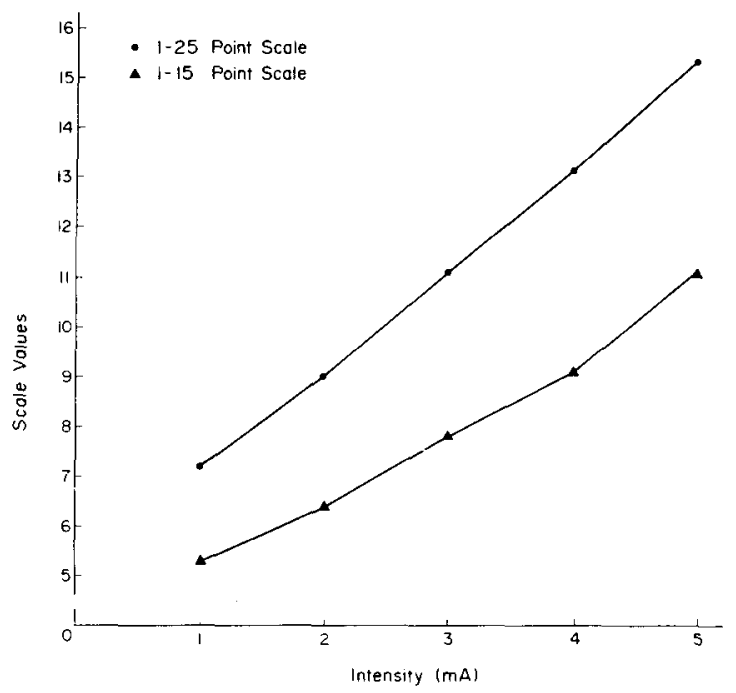

Figure 4. Psychophysical functions for the 1-15 point scale and the 1-25 point scale (Experiment 2).
Table 2

Rate Parameters (b) and Goodness-of-Fit Statistics, $\mathbf{r}^{2}$, for the Four Psychophysical Functions From the Data Obtained in the 1-15- and 1-25-Point Rating-Scale Experiments (Experiment 2)

\begin{tabular}{|c|c|c|c|c|c|}
\hline \multirow[b]{3}{*}{$\begin{array}{l}\text { Rating } \\
\text { Scale }\end{array}$} & & \multicolumn{4}{|c|}{ Psychophysical Functions } \\
\hline & & \multirow[b]{2}{*}{ Linear } & \multirow[b]{2}{*}{$\mathrm{I}^{2}$} & \multicolumn{2}{|c|}{ Power } \\
\hline & & & & $\begin{array}{c}\text { Two- } \\
\text { Parameter }\end{array}$ & $\begin{array}{c}\text { Three- } \\
\text { Parameter }\end{array}$ \\
\hline $1-15$ & $\begin{array}{l}b \\
r^{2}\end{array}$ & $\begin{array}{r}1.22 \\
.9986\end{array}$ & $\begin{array}{r}0.20 \\
.9690\end{array}$ & $\begin{array}{r}0.40 \\
.9732\end{array}$ & $\begin{array}{r}1.11 \\
.9988\end{array}$ \\
\hline $1-25$ & $\begin{array}{l}b \\
r^{2}\end{array}$ & $\begin{array}{r}2.03 \\
.9985\end{array}$ & $\begin{array}{r}0.33 \\
.9749\end{array}$ & $\begin{array}{r}0.46 \\
.9726\end{array}$ & $\begin{array}{r}1.17 \\
.9996\end{array}$ \\
\hline
\end{tabular}

Note-The functions were fit as in Experiment 1.

( 3 times the modulus) or 900 (9 times the modulus). The psychophysical function was appreciably steeper in the latter condition (see also Robinson, 1976). The present effect seems predictable from Parducci's (1974) range-frequency theory, which essentially presumes that psychophysical judgments reflect two types of response bias, a tendency to partition the stimulus range into equal parts, and a tendency to use all categories with equal frequency. The second tendency would account for the observed change in the slopes of the psychophysical functions in the present experiment.

For the 1-15-point condition, we again obtained evidence of a recency effect with a value of $w$ equal to .36-a recency effect in serial judgment that is often thought to be characteristic of psychophysical tasks (see Anderson, 1981, p. 154). However, for the 1-25-point condition, we found slight evidence for a primary effect $(w=.54)$. The determining factors of the value of $w$ are not well understood (although see Anderson, 1981, pp. 271-273). In the following experiment it will be shown that the value of $w$ is associated to some extent with the perceived painfulness of the stimuli (cf. Jones, 1980).

\section{EXPERIMENT 3}

If we can assume that the subjective magnitudes of the five intensities are linearly spaced, what implications might this have for the evaluation of analgesic procedures? The rationale of the present experiment is based upon a determination of psychological and psychophysical rules in subjects who differ in the degree to which they report the same shock intensities as eliciting pain. Variation between people in such measures as pain threshold (the stimulus magnitude for which a subject first reports feeling pain) and tolerance (the most extreme stimulus magnitude that the subject is willing or able to experience) is typically quite high (see Clark \& Bindra, 1956; Clausen \& King, 1950; Dillon, 1968; Notermans \& Tophof, 1967; 
Wolff \& Jarvik, 1965). Moreover, reliable differences in performance have been obtained between subjects who rated stimuli as highly painful and those who rated the same stimuli as less painful (Jones, Planas, \& Anuza, 1982). Hence, it may be possible to demonstrate differences in how subjects combine and scale the same stimuli according to the degree to which they experience the stimuli as painful. Judgments by subjects who experienced stimulus intensities as less painful would then provide a convenient baseline for determining the effectiveness of an analgesic.

\section{Method}

Subjects. The subjects were 27 first-year undergraduates not tested in Experiments 1 and 2. They also participated for course credit. As before, the subjects were free to withdraw from the experiment without loss of credit.

Apparatus and Procedure. The apparatus was the equipment used in the first two experiments. The first phase of the experiment followed precisely the procedure of Experiment 1; that is, the combined intensity of shocks was rated on an anchored 1-20point scale. Immediately following this phase, the subjects were presented with a series of single shocks, each $500 \mathrm{msec}$ in duration, ranging in intensity from $.10 \mathrm{~mA}$ through $.20, .30, .40, .50, .60$, $.70, .80, .90,1,2,3,4$, and $5 \mathrm{~mA}$. The subject was asked to rate the shocks on a scale from 0 to 10 . The points $0,1,5$ and 10 were labeled, respectively, "Can't feel anything," "Just detectable intensity," "Just noticeably painful," and "Intolerably painful.", In this way, the subject's experience of the painfulness of the shocks could be assessed.

\section{Results}

It is convenient to deal first with the pain ratings obtained in the second phase of the experiment. The subjects were divided into two groups on the basis of their mean responses to the maximum-intensity 5-mA shock. Twelve subjects ( 8 males and 4 females) gave mean ratings greater than 9 (higher group) and 15 subjects ( 13 males and 2 females) gave mean ratings less than 9. We have noted the numbers of males and females in each group because our research has shown that females typically rate the same shock intensities as more painful than do males (Jones \& Gwynn, 1983).

The mean ratings for each group as a function of shock intensity are shown in Figure 5 . It is clear that the two curves diverge at about $0.20 \mathrm{~mA}$ and analysis of variance showed a highly significant difference between the groups $[F(1,25)=24.14, p<.001]$. The effect of intensity was also highly significant $[F(13$, $325)=374.57, \mathrm{p}<.001$ ], as was the two-way interaction $[F(13,325)=11.87, \mathrm{p}<.001]$. A more detailed examination of the subjects' ratings of the shocks as painful is shown in Figure 6. The upper panel is a plot of the mean probability of responding at least 5, "Just noticeably painful," and the lower panel plots the probability of responding 10 , "Intolerably painful." There was a probability of .50 that the higher groups would report just noticeable pain at $.9 \mathrm{~mA}$. The same probability was achieved by the

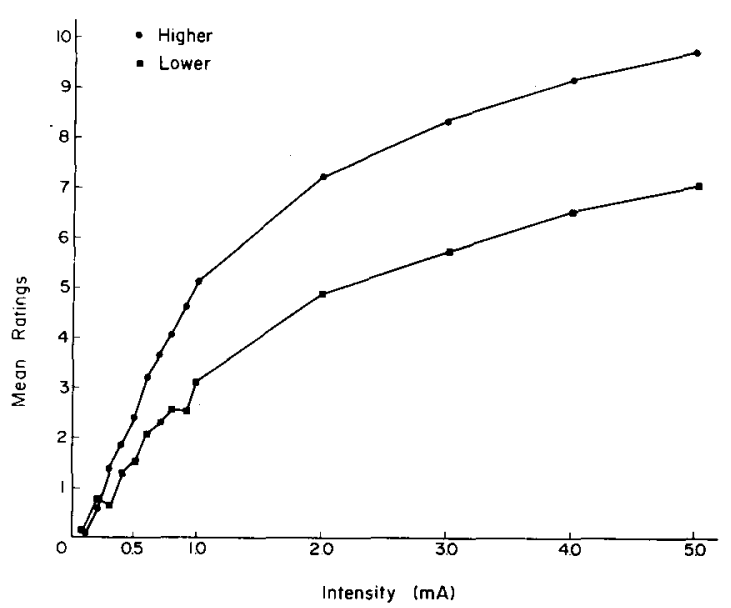

Figure 5. Mean ratings of single shocks by higher and lower pain raters (Experiment 3).

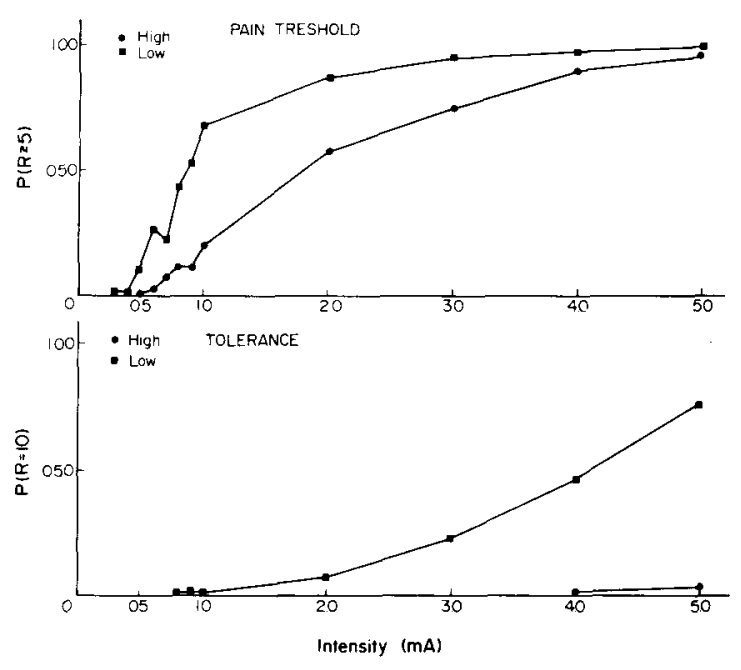

Figure 6. Mean probability of responding at least $5(p>5)$, “Just noticeable pain" (upper panel) and at least $10(p=10)$, "Intolerable pain" (lower panel) for higher and lower pain raters (Experiment 3).

lower group for an intensity twice as high (1.8 mA). The lower panel of Figure 6 reveals even greater differences. The higher group were 23 times more likely than the lower group to judge the 5-mA shock as intolerably painful. The modal probability of responding 10 to the 5-mA shock in the higher group was unity $(n=5)$; the modal probability was zero $(n=13)$ for the lower group. The probability of a subject in the higher group responding 10 to the 5-mA shock was never less than .5 , whereas the same probability reached $\mathbf{0 . 2 5}$ for only two of the lower group.

Mean ratings for the factorially paired stimuli are shown in Figure 7 for the two groups. Both sets of curves appear to be reasonably linear and parallel. Analysis of variance for higher raters indicated significant main effects of rows $[F(4,44)=24.21$, $p<$ $.001]$ and columns $[F(4,44)=42.29, p<.001]$ and 
the absence of a significant interaction $[F(16,176)<1]$. The analysis of variance for lower raters gave much the same results [rows, $F(4,56)=27.18, p<.001$; columns, $F(4,56)=46.53, \mathrm{p}<.001$; and the interaction, $F(16,224)=1.08, p=.38$ ]. In short, Equation 3 is a reasonable model of the integration process for both higher and lower raters.

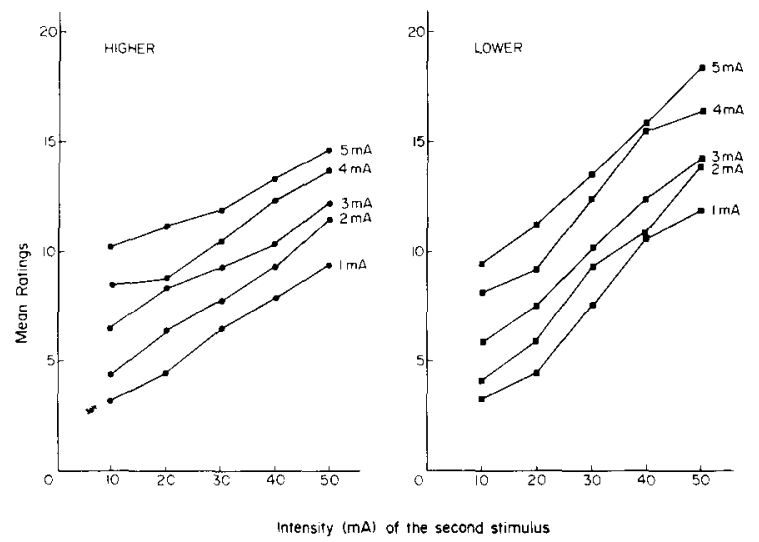

Figure 7. Mean ratings of the second stimulus as a function of the first for higher pain raters (left-hand panel) and lower pain raters (right-hand panel) (Experiment 3).

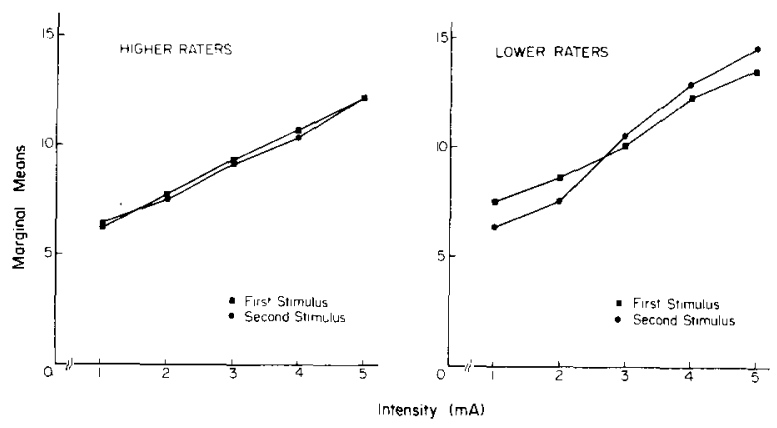

Figure 8. Marginal means for the first and second stimuli illustrating the equivalent weighting of stimuli by higher pain raters (left-hand panel) and the greater weighting of the second stimulus by lower pain raters (right-hand panel) (Experiment 3).

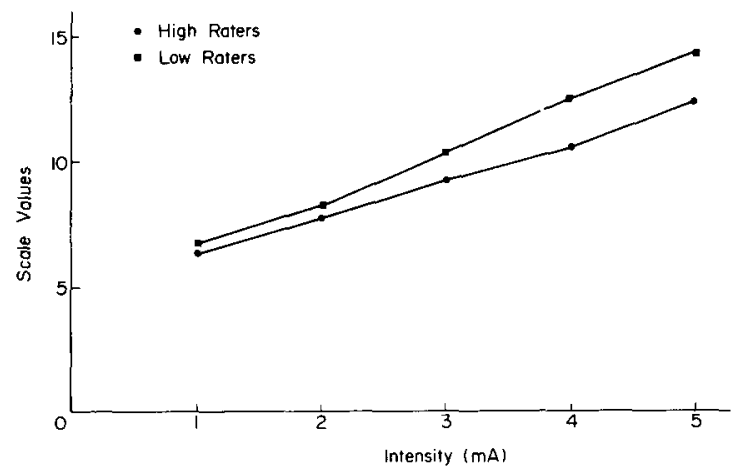

Figure 9. Psychophysical functions derived from the ratings in Figure 7 for higher and lower pain raters (Experiment 3).
Table 3

Rate Parameters (b) and Goodness-to-Fit Statistics $\left(r^{2}\right)$ for the Four Psychophysical Functions Obtained From the Data from Higher and Lower Raters (Experiment 3)

\begin{tabular}{ccrrrr} 
& & \multicolumn{3}{c}{ Psychophysical Functions } \\
\cline { 3 - 6 } & & & & \multicolumn{2}{c}{ Power } \\
\cline { 3 - 6 } Ratios & & Linear & \multicolumn{1}{c}{$\mathrm{I}^{2}$} & $\begin{array}{c}\text { Two- } \\
\text { Parameter }\end{array}$ & $\begin{array}{c}\text { Three- } \\
\text { Parameter }\end{array}$ \\
\hline \multirow{2}{*}{ Higher } & $\mathrm{b}$ & 1.45 & 0.23 & 0.39 & 1.05 \\
& $\mathrm{r}^{2}$ & .9997 & .9670 & .9783 & .9996 \\
& $\mathrm{~b}$ & 1.88 & 0.30 & 0.45 & 1.17 \\
Lower & $\mathrm{r}^{2}$ & .9905 & .9647 & .9546 & .9716 \\
\hline
\end{tabular}

Note-The functions were fit as in Experiments 1 and 2 .

Figure 8, however, shows that the value of the weight parameter tends to differentiate the two groups. The figure is a plot of the marginal means for the first (rows) and second (columns) stimuli, with higher raters in the left-hand panel and lower raters in the right-hand panel. For the higher group, the two curves are essentially the same, whereas for the lower raters, the curve for the second stimuli was steeper. Since $w$ is proportional to the slope of the row marginals (Anderson, 1974), Figure 8 shows that the higher raters accorded an equal weight to both stimuli and the lower raters accorded a greater weight (.59) to the second stimulus.

Psychophysical functions for the two groups are shown in Figure 9. Both curves appear linear, and Table 3 shows that a linear rule is superior to the power fits and to the $I^{2}$ rule for both subject groups. The slope of the psychophysical function for higher raters is $20 \%$ lower than the slope for lower raters. As a statistical test of the reliability of the difference between the slopes, values of the regression coefficient were computed (least squares) for each subject, and a t test showed that the values in the higher group were significantly lower $[\mathrm{t}(25)=2.28, \mathrm{p}<.005]$.

\section{DISCUSSION}

The first two experiments show that the linear spacing of the five stimuli was not affected by the number of available rating categories, at least within the range of categories used in these experiments. The slope of the psychophysical function did, however, depend lawfully upon the context; the greater the number of categories, the steeper the curve. Rangefrequency theory, which adequately predicts scale values obtained from ratings of single stimuli, appears also to predict the values obtained here for judgments of the combined value of two stimuli.

The fact that we were unable to confirm the form of the psychophysical function obtained by Jones (1980) need occasion no surprise unless we presume, contrary to a good deal of evidence, that such func- 
tions reflect no more than the rate of transduction of physical intensities by a particular sensory system. Psychophysical judgments are clearly and inevitably influenced by many factors, of which the immediately present stimulus (or stimuli) is only one (cf. Anderson, 1975, 1981; Birnbaum, 1978; Mellers \& Birnbaum, 1982; Parducci, 1974; Poulton, 1967, 1979; Robinson, 1976).

More importantly, we have confirmed that the weighted averaging model (Equation 3 ) provides an appropriate integration rule for five distinct data sets and does not depend upon the number of rating categories or upon whether subjects experience the shocks as more or less painful. Validation of the parallelism postulate implicit in Equation 3 for judgments of painful stimuli has important consequences for the psychological representation of stimuli. Parallelism of lines constitutes an equivalence relation (determinate, reflexive, symmetric, and transitive), and therefore provides a simple, even elementary, representation. Holman and Marley (1974) have noted that the conditions for equivalence relations are ordinarily difficult to meet in practice, so that these results for stimuli that might reasonably be expected to lead to violations of equivalence are particularly encouraging.

An emphasis on psychological laws such as Equation 3 has a certain conceptual plausibility in the study of pain. It is generally accepted that noxious stimuli elicit complex, emotionally colored responses (e.g., Tursky, 1974). The experience of pain depends upon the meaning or the interpretation of stimuli as well as upon their physical properties (Beecher, 1959). Because the weighting process reflects the relative evaluation of stimuli, estimation of $w$ may provide a flexible means of determining quantitatively how important or how focal a particular stimulus is taken to be.

Although Equation 3 provided an equivalent fit for higher and lower raters in Experiment 3, the value of the weight parameter, at least when estimated for groups, differed in the two cases. Higher raters accorded equivalent weight to both stimuli, but a recency effect (higher weighting of $s_{j}$ ) was obtained for subjects who reported lower pain. Jones (1980) found analogous results for higher and lower intensity ranges. The weighting of $s_{i}$ and $s_{j}$ was roughly equivalent for the higher range, and $s_{\mathrm{j}}$ received a higher weighting for the lower range. The value of $w=.42$ obtained in the previous study for the range .60 to $.90 \mathrm{~mA}$ was essentially the same as the value obtained here for the range 1 to $5 \mathrm{~mA}$ provided that subjects did not experience these intensities as maximally painful. Jones (1980) argued that the equal weighting of the stimuli reflected their equal salience. Both stimuli are qualitatively painful and may be given equal attention by higher raters. Judgments by lower raters are on average less influenced by the painfulness of the stimuli, and we observe the recency effect typical of psychophysical judgment.

Jones (1980) suggested that the good fit obtained for Equation 4 (the $1^{2}$ model) for stimuli in the higher range supported Rollman's (1969) contention that electrical stimuli bypass cutaneous receptors and directly excite afferent nerves. This argument assumed a generality for the $I^{2}$ model that, in light of the present results, it does possess. Of course, this is not to say that Rollman's hypothesis has no support-merely that it is not supported by the finding that psychophysical functions for electric shock are positively accelerated.

The linear functions we obtained did differentiate higher and lower raters. The rate of increase in scale values as a function of stimulus intensities was relatively greater for lower raters. This corresponds to Sternbach and Tursky's finding that the exponents estimated for Equation 1 were lower when the stimulus range included painful stimuli (the fact that they obtained power functions may reflect no more than a nonlinear bias introduced by magnitude estimation). There are a number of possible explanations for these results. The first appears specific to electric shock experiments. As the painfulness of shocks increases, the subject's ability to discriminate intensities deteriorates markedly (Jones et al., 1982; Jones \& Gwynn, 1983). The steeper slope of the psychophysical function for higher raters may therefore reflect a somewhat greater ability on their part to discriminate the stimuli either because they experience less pain or because they do not focus on the painfulness of stimuli to the same extent as lower raters. A second explanation is based on the range-frequency theory. It is commonly found that steeper psychophysical functions are associated with narrower stimulus ranges. Poulton (1967) appears to have been the first to notice the negative correlation between stimulus range and the exponent of Equation 1 for magnitude estimates (cf. Teghtsoonian, 1971). Mellers and Birnbaum (1982) have also found that scale values derived from ratings increase more rapidly for a medium range of stimuli than for a wide range. One may speculate that the "stimulus range" is, to some extent, a subject variable. The same range of physical intensities may be wider for the higher raters than for the lower raters because the range of painful experiences is, in fact, wider for this group. The steeper curve for the higher raters would then simply be a function of the stimulus range, although the stimulus range is now defined by both physical intensity and subjective experience.

Both accounts are, of course, highly speculative. The point to notice here is that, whatever the case, an increase in the slope of the psychophysical function together with a decrease in the weighting of the first stimulus relative to the second may provide a model for the evaluation of any analgesic effect. Of 
course, algebraic models such as Equation 3 do not lead to any routine scaling procedure. It is an empirical question whether or not the integration model is valid, and we have suggested that the integration process may break down if stimuli are experienced as much more painful. However, if the model is appropriate, a valid representation of stimuli is possible, and it is a simple matter to derive scales of subjective magnitude.

\section{REFERENCES}

Anderson, N. H. (1974). Algebraic models in perception. In E. C. Carterette \& M. P. Friedman (Eds.), Handbook of perception (Vol. 2). New York: Academic Press.

Ande rson, N. H. (1975). On the role of context effects in psychophysical judgment. Psychological Review, 82, 462-482.

Anderson, N. H. (1981). Foundations of information integration theory. New York: Academic Press.

Beck, C., \& Rosner, B. S. (1968). Magnitude scales and somatic evoked potentials to percutaneous electric stimulation. Psychophysiology and Behavior, 3, 949-983.

BEECHER, H. K. (1959). Measurement of subjective responses. New York: Oxford University Press.

Birnbaum, M. H. (1978). Differences and ratios in psychophysical measurement. In N. J. Castellan \& F. Restle (Eds.), Cognitive theory (Vol. 3). Hillsdale, $\mathrm{NJ}$ : Erlbaum.

Clark, J. W., \& Bindra, D. (1956). Individual differences in pain thresholds. Canadian Journal of Psychology, 10, 69-76.

Clausen, J., \& King, H. E. (1950). Determination of the pain threshold in untrained subjects. Journal of Psychology, 30, 299-306.

Cross, D. V., Tursky, B., \& Lodge, M. (1975). The role of regression and range effects in determination of the power function for electric shock. Perception \& Psychophysics, 18, 9-14.

Dillon, D. J. (1968). Stimulus intensity, site of stimulation, and individual reactivity as determinants of the energy threshold for pricking pain. Journal of Experimental Psychology, 77, 559-566.

Ekman, G., Frankenhaeuser, M., Levander, S., \& Mellis, I. (1964). Scales of unpleasantness of electrical stimulation. Scandinavian Journal of Psychology, 5, 257-261.

Gibson, R. H., \& Tomko, D. L. (1972). The relation between category and magnitude estimates of tactile intensity. Perception \& Psychophysics, 12, 135-138.

Gracely, R. H., McGrath, P., \& Dubner, R. (1978). Ratio scales of sensory and affective verbal pain descriptors. Pain, 5, 5-18.

Holman, E. W., \& Marley, A. A. J. (1974). Stimulus and response measurement. In E. C. Carterette \& M. P. Friedman (Eds.), Handbook of perception (Vol. 2). New York: Academic Press.

JoNES, B. (1980). Algebraic models for integration of painful and nonpainful electric shocks. Perception \& Psychophysics, 28, 572-576.

Jones, B., \& GwYnn, M. (1983). Sex differences in response to painful electric shocks. Manuscript submitted for publication.

Jones, B., Planas, M., \& Anuza, T. (1982). Painfulness de- creases the discriminability of electric shock. Perception \& Psychophysics, 32, 187-191.

McCallum, P., \& Goldberg, H. (1975). Magnitude scales for electrocutaneous stimulation. Perception \& Psychophysics, 17, 75-78.

MarquardT, D. W. (1964). An algorithm for least-squares estimation of non-linear parameters. Journal of the Society for Industrial and Applied Mathematics, 2, 431-441.

Melle Rs, B. A., \& Birnbaum, M. H. (1982). Loci of contextual effects in judgment. Journal of Experimental Psychology: Human Perception and Performance, 8, 582-601.

Notermans, S. L. H., \& Tophof, M. M. W. A. (1967). Sex differences in pain tolerance and pain apperception. Psychiatra, Neurologia, Neurochirurgia, 70, 23-29.

Parducci, A. (1974). Contextual effects: A range frequency analysis. In E. C. Carterette \& M. P. Friedman (Eds.), Handbook of perception (Vol. 2). New York: Academic Press.

Poulton, E. C. (1967). Population norms of top sensory magnitudes and S. S. Stevens exponents. Perception \& Psychophysics, 2, 312-316.

Poulton, E. C. (1979). Models for biases in judging sensory magnitude. Psychological Bulletin, 86, 777-803.

Robinson, G. E. (1976). Biasing power law exponents in magnitude estimation instructions. Perception \& Psychophysics, 19, 80-84.

Rollman, G. E. (1969). Electrocutaneous stimulation: Psychometric functions and temporal integration. Perception \& Psychophysics, 5, 289-293.

She PARD, R. N. (1981). Psychological relations and psychophysical scales: On the basis of direct psychological measurement. Journal of Mathematical Psychology, 24, 21-57.

Stam, H. J., Petrusic, W. M., \& Spanos, N. P. (1981). Magnitude scales for cold pressor pain. Perception \& Psychophysics, 29, 612-617.

Sternbach, R. A., \& Tursky, B. (1964). On the psychophysical power function in electric shock. Psychonomic Science, 1, 217 218.

Stevens, S. S. (1957). On the psychophysical law. Psychological Review, 64, 153-181.

STEvens, S. S. (1966). Matching functions between loudness and ten other continua. Perception \& Psychophysics, 1, 5-8.

Stevens, S. S. (1975). Psychophysics. New York: Wiley.

Stevens, S. S., Carton, A. S., \& Shickman, G. M. (1958). A scale of apparent intensity of electric shock. Journal of Experimental Psychology, 56, 328-334.

Teghtsoonian, R. (1971). On the exponent in Stevens' law and the constant in Ekman's law. Psychological Review, 78, 71-80.

Tursky, B. (1974). Physical, physiological, and psychological factors that affect pain reaction to electric shock. Psychophysiology, 11, 95-112.

Tursky, B., Watson, P. D., \& O'Connell, D. N. (1965). A concentric shock electrode for pain stimulation. Psychophysiology, 1, 269-298.

WolfF, B. B., \& JARvik, M. E. (1965). Quantitative measures of deep somatic pain: Further studies with hypertonic saline. Clinical Science, 28, 43-56.

(Manuscript received January 18, 1983; revision accepted for publication September 30, 1983.) 